\begin{tabular}{|c|c|c|c|c|}
\hline MADIT-ICD Benefit Group & Highest & Intermediate & Lowest & $p$ value \\
\hline $\begin{array}{l}\text { At Baseline } \\
\mathrm{n}=103(\%)\end{array}$ & $\begin{array}{l}31 \\
(30.1)\end{array}$ & $61(59.2)$ & $\begin{array}{l}11 \\
(10.7)\end{array}$ & $\begin{array}{l}p<0.001 \mathrm{H} \\
\text { vs I } \\
p<0.006 \mathrm{H} \\
\text { vs } L \\
p<0.001 \text { I } \\
\text { Vs } L\end{array}$ \\
\hline VT $/ V F$ risk score [Mean $\pm S D$ ] & $\begin{array}{l}8.3 \pm \\
1.5\end{array}$ & $7.7 \pm 2.1$ & $5 \pm 1.3$ & \\
\hline NAM risk score $[$ Mean \pm SD] & $\begin{array}{l}1.1 \pm \\
0.8\end{array}$ & $3.3 \pm 1.8$ & $\begin{array}{l}4.8 \pm \\
1.3\end{array}$ & \\
\hline $\begin{array}{l}\text { On Follow-Up: } 1642 \text { days } \pm 535 \text {, } \\
\text { [1727], (17-2528). }\end{array}$ & & & & \\
\hline $\begin{array}{l}\text { Significant VT/NF } \mathrm{n}=20(\%) \\
\text { [\% of benefit group] }\end{array}$ & $\begin{array}{l}7 \text { (35) } \\
{[22.6]}\end{array}$ & $\begin{array}{l}12(60) \\
{[19.7]}\end{array}$ & $\begin{array}{l}1(5) \\
{[9.1]}\end{array}$ & $\begin{array}{l}p=N S H \text { vs } \\
\text { I } \\
p<0.02 H \\
\text { vs } L \\
p<0.002 \text { I } \\
\text { vs } L\end{array}$ \\
\hline Non-Arrhythmic Mortality $\mathrm{n}=11(\%)$ & $3(27.3)$ & $7(63.6)$ & $1(9.1)$ & $\mathrm{p}=\mathrm{NS} H$ vs \\
\hline [\% of benefit group] & [9.7] & {$[11.5]$} & [9.1] & $\begin{array}{l}\text { I } \\
p=N S H \text { vs } \\
L \\
p=0.009 \text { I } \\
\text { vs } L \\
\text { [p=NS H vs } \\
\text { I } \\
p=N S \text { H vs } \\
\text { L } \\
p=N S ~ L \text { vs } \\
\text { I] }\end{array}$ \\
\hline No events $\mathrm{n}=72(\%)$ & 21 & $42(58.3)$ & 9 & [ $p=N S$ H vs \\
\hline [\% of benefit group] & $\begin{array}{l}(29.2) \\
{[67.7]}\end{array}$ & [68.8] & $\begin{array}{l}(12.5) \\
{[81.8]}\end{array}$ & $\begin{array}{l}\text { I } \\
p=N S \text { H vs } \\
\text { L } \\
p=N S \text { I vs } \\
\text { L] }\end{array}$ \\
\hline
\end{tabular}

Table $A$ (Legend: $H=H i g h, I=$ Intermediate, $L=$ Lowest)

Results (Table 1A) Whole cohort: Age: $64 \pm 11$, [67], (3589); Female: 27/103 (26.2\%); CRT-D: 35 (34\%); Diabetes: 24 (23.3\%); Atrial Arrhythmia: 22 (21.4\%); Previous Myocardial Infarction: 68 (66\%); NSVT: 22 (21.4\%). At baseline, significant majority were in the Highest or Intermediate BG. On follow-up, the same BG's had a majority of VT/VF events. Moreover, the proportion of VT/VF within each BG corresponded to the grade of benefit: Highest (22.6\%), Intermediate $(19.7 \%)$, Lowest $(9.1 \%)$. The distribution of NAM was less well defined, and spanned across all BG's, with the majority occurring in the Intermediate BG. No significant differences among the BG's were seen in for patients with no events. Conclusion In our experience, over the medium term $(\sim 5$ years), the MIBPS was best able to suggest candidates with a high (High BG) or a low (Low BG) likelihood of benefit from a primary prevention ICD. It was not able to as clearly define those likely to suffer NAM, irrespective of the BG. Likely better tools are required to determine the benefit of an ICD in the Intermediate BG.

Conflict of Interest Nil

\section{OPTIMAL LEFT VENTRICULAR LEAD POSITIONING DURING CARDIAC RESYNCHRONISATION THERAPY; A COMPARISON OF 2 METHODS}

${ }^{1}$ Panagiota Anna Chousou, ${ }^{2}$ Rahul Chattopadhyay, ${ }^{2}$ Katie Sanders, ${ }^{2}$ Viki Carpenter, 'Julie Hayes, ${ }^{3}$ Vassilios Vassiliou, 'Peter J Pugh. ' Cambridge University Hospital, Cambridge, UK; ${ }^{2}$ Addenbrooke's Hospital, Cambridge University Hospitals NHS Foundation Trust; ${ }^{3}$ Norwich Medical School, University of East Anglia

\subsection{6/heartjnl-2021-BCS.90}

Background/Introduction Superior response to CRT and improved clinical outcomes can be achieved when placing the left ventricular (LV) lead at the site of latest mechanical activation and avoiding regions of scar during cardiac resynchronisation therapy (CRT). The site of latest mechanical activation can be identified using speckle-tracking echocardiography according to the TARGET study, whilst the area of latest electrical activation can be assessed by measuring the intrinsic LV electric delay (QLV), which is the time from the initial deflection of the surface QRS complex to sensing at the LV lead electrode. It remains unclear if the two techniques are interchangeable and whether the site of latest mechanical activation is related to the site of latest electrical activation, or whether sensed electrical signals correspond to sites of scar.Purpose: The purpose of this study was to determine whether electrical targeting of LV lead position corresponds to echo-guided mechanical targeting and whether myocardial electrogram signals can predict areas of myocardial scar

Methods This was a pilot study of patients receiving CRT, in sinus rhythm with severe LV impairment (ejection fraction $\leq 35 \%$ ) and left bundle branch block (LBBB) with QRS $\geq 130 \mathrm{~ms}$. Ethics approval was obtained, and written consent was sought. Participants underwent echocardiographic speckletracking 2-dimensional radial strain imaging to identify amplitude and time to peak contraction of posterior, posterolateral, lateral, anterolateral and anterior segments. During CRT implant, the sensed R wave and QLV were measured at the respective branch of the coronary sinus, where the anatomy permitted. The site of latest mechanical was compared to the site of latest electrical activation and classified as concordant (same site), adjacent (within 1 segment), or remote (2 segments away). The strain amplitude was compared to the sensed $\mathrm{R}$ wave and time to mechanical activation with QLV.

Results Seventeen patients (13 male) were studied, mean age 74.2 (SD 8.7). Mean QRS was 161 ms (SD 18), mean PR $186 \mathrm{~ms}$ (SD 37). A modest and significant positive correlation was found between the site of latest mechanical and latest electrical activation (Pearson $\mathrm{r}=0.66, \mathrm{p}$ 0.004). The site of latest electrical activation was concordant with latest mechanical activation in 13 patients (76.5\%), adjacent in 4 patients $(23.5 \%)$ and remote in none. There was no apparent association between sensed $\mathrm{R}$ wave and strain amplitude (Pearson $\mathrm{r}=0.2, \mathrm{p}=0.12)$ or between QLV and time to mechanical activation (Pearson $\mathrm{r}=0.1, \mathrm{p}=0.5$ ).

Conclusion A significant positive correlation was found between the site of latest mechanical and latest electrical activation in patients undergoing CRT implant. Electrical targeting might be a suitable alternative to mechanical targeting particularly when imaging is not available, however, does not appear to discriminate scar. Future larger studies are needed to confirm our findings and determine whether QLV can be used as an alternative method for targeting LV lead placement.

Conflict of Interest None 